\title{
Operator lifetime and the force-free electrodynamic limit of magnetised holographic plasma
}

\author{
Napat Poovuttikul ${ }^{a, b}$ and Aruna Rajagopal ${ }^{b}$ \\ ${ }^{a}$ Department of Mathematical Sciences, Durham University, \\ South Road, Durham DH1 3LE, U.K. \\ ${ }^{b}$ Science Institute, University of Iceland, \\ Dunhaga 3, IS-10\%, Reykjavik, Iceland \\ E-mail: napat.poovuttikul@durham.ac.uk, arr17@hi.is
}

ABSTRACT: Using the framework of higher-form global symmetries, we examine the regime of validity of force-free electrodynamics by evaluating the lifetime of the electric field operator, which is non-conserved due to screening effects. We focus on a holographic model which has the same global symmetry as that of low energy plasma and obtain the lifetime of (non-conserved) electric flux in a strong magnetic field regime. The lifetime is inversely correlated to the magnetic field strength and thus suppressed in the strong field regime.

KEYwORDS: AdS-CFT Correspondence, Effective Field Theories

ArXiv EPrint: 2101.12540 


\section{Contents}

1 Introduction 1

2 The holographic model 4

2.1 Linearised solutions in $\omega / T \ll 1$ limit and matching procedure $\quad 6$

2.1.1 Perturbation parallel to equilibrium magnetic field 6

2.1.2 Perturbation perpendicular to equilibrium magnetic field 8

2.2 Checking $T \gtrsim 0$ limit in $\omega / \sqrt{|\mathbf{B}|} \ll 1$ regime $\quad 11$

2.2.1 Zero temperature 11

2.2.2 $T \lesssim \omega \ll \sqrt{|\mathbf{B}|}$ limit 12

3 Conclusion $\quad 14$

$\begin{array}{ll}\text { A Numerical solution and evaluation of operators lifetime } & 15\end{array}$

$\begin{array}{ll}\text { B Frobenius analysis in } A d S_{3} \times \mathbb{R}^{2} \text { region } & 16\end{array}$

\section{Introduction}

Hydrodynamics [1] is a well-established theoretical framework which universally describes the long wavelength, low frequency behaviour of interacting systems at finite temperature. Essentially, hydrodynamic theory is a description of conserved quantities and the manifestation of the corresponding symmetries in a system in thermal equilibrium. Theories with widely varying microscopics can have the same macroscopic hydrodynamic description. One possible explanation why such a universal description is possible is that all operators except conserved charges have parametrically short lifetimes compared to the scale of interest and, once the longest-lived non-conserved operator ${ }^{1}$ has decayed away, the hydrodynamic description becomes viable (see figure 1).

The hydrodynamic framework may be generalised to systems where the conserved charges are those of a higher-form symmetry [5] which counts the number density of extended objects. A recent exploration of this idea [6] (see also [7-10]) shows that the resulting hydrodynamics of a one-form $\mathrm{U}(1)$ charge reproduces the theory of magnetohydrodynamics (MHD). ${ }^{2}$ This should not come as a big surprise. MHD is, after all, a low energy effective theory of plasma where the (dynamical) electric field is screened - the one-form U(1) symmetry associated to electric flux is explicitly broken. This implies that in, for example,

\footnotetext{
${ }^{1}$ While this operator language is more familiar in the context of quantum systems, it is also applicable to classical systems via e.g. memory matrix formalism [2, 3]. A more modern introduction may be found in [4].

${ }^{2}$ For the formulation of MHD that closely resembles higher-form symmetry formulation, see e.g. [11-13].
} 


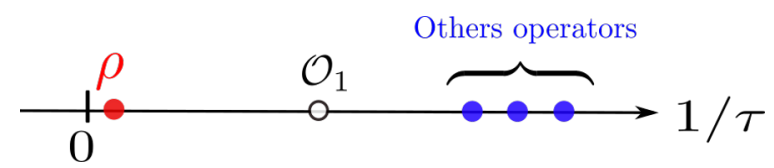

Figure 1. A cartoon illustration of the lifetime of operators of a theory that exhibit hydrodynamic behaviour at late time. Here, there is a parametrically large gap between conserved charges $\rho$ and the rest. The life time $\tau_{1}$ of the longest-lived non-conserved operator, denoted by $\mathcal{O}_{1}$ set the time scale in which hydrodynamics becomes applicable.

a plasma at zero magnetic field (where the Ohm's law $\mathbf{j}=\sigma \mathbf{E}$ is a good approximation) the electric field has a finite lifetime,

$$
\delta \mathbf{E} \propto \exp \left(-t / \tau_{E}\right) \quad \Longleftrightarrow \quad\left\langle E^{i}(-\omega) E^{j}(\omega)\right\rangle \sim \frac{\delta^{i j}}{\omega+i / \tau_{E}},
$$

with the lifetime of the electric field $\tau_{E}=1 / \sigma$. The conductivity $\sigma$ can be computed from first principles. For instance, quantum electrodynamics at weak coupling, it can be written as $[14]^{3}$

$$
\sigma \propto \frac{T}{e^{2} \log e^{-1}},
$$

where $e$ is the electromagnetic coupling. The lifetime of electric field $\tau \sim 1 / T$, is then much shorter than the scale $t \gg 1 / T$ (or $\omega / T \ll 1$ in Fourier space) where hydrodynamic behaviour is expected. If, in this late-time limit, all other operators except energy density $T^{t t}$ and the momentum $T^{t i}$ have already decayed away, one can expect the hydrodynamic description of a plasma to be governed by

$$
\partial_{\mu} T^{\mu \nu}=0, \quad \partial_{\mu} J^{\mu \nu}=0 .
$$

The conserved currents $T^{\mu \nu}$ and $J^{\mu \nu}$ are expressed in terms of energy, momentum, magnetic flux $J^{t i} \equiv B^{i}$ and their conjugates, organised order by order in the gradient expansion. This formulation of MHD only requires macroscopic consistency and does not require the introduction of the gauge field $\star J=F=d A$ which, due to screening effect, is not a long-lived degree of freedom.

This brings us to the central question of the present paper: is a hydrodynamic description of the form (1.3) applicable in the limit low temperature compared to magnetic flux density $T^{2} /|\mathbf{B}| \ll 1$ ? This question is important if one wants to apply the MHD description to astrophysical plasmas where the magnetic field is many orders of magnitude larger than the scale set by the temperature. ${ }^{4}$ If one were to naively extrapolate (1.1)-(1.2), the lifetime of the electric field appears to become arbitrarily long as the temperature decreases. However, there exists a macroscopic description of plasma in this regime that

\footnotetext{
${ }^{3}$ The fact that this quantity has only been computed at the beginning of this century indicates the difficulty of the required computations.

${ }^{4}$ To get some intuition of the ratio between $T / \sqrt{B}$ in the setup with astrophysical interest, let us consider the magnetosphere of the pulsar. Here, the typical magnetic field strengh is $\approx 10^{8}$ Tesla (or $\sim 10^{11} \mathrm{eV}^{2}$ in natural unit), see e.g. [15] and reference therein. On the other hand, temperature can be generously estimated to be of range $10^{0}-10^{2} \mathrm{~K}$ ( or $10^{-5}-10^{-3} \mathrm{eV}$ in the natural unit).
} 
has been successfully applied. This theory is called force-free electrodynamics or FFE, and has been used extensively in astrophysical setups such as in the magnetosphere of black holes [16, 17], neutron star [18] and solar corona [19] just to name a few. In its conventional form, this theory is applied to a system which is magnetically dominated (i.e. $\left|\mathbf{B}^{2}\right|>\left|\mathbf{E}^{2}\right|$ or, covariantly $F_{\mu \nu} F^{\mu \nu}>0$ ) and whose dynamics is governed by

$$
\begin{aligned}
\epsilon^{\mu \nu \rho \sigma} F_{\mu \nu} F_{\rho \sigma} & =0, \\
F^{\mu \nu} \nabla_{\lambda} F^{\lambda} & =0 .
\end{aligned}
$$

Here, the first relation implies that $\mathbf{E} \cdot \mathbf{B}=0$ while the second relation implies that the force $j_{e l}^{\mu} F_{\mu \nu}$, with $j_{e l}^{\mu}:=\nabla_{\nu} F^{\nu \mu}$ via Maxwell's equations, acting on plasma vanishes (hence the name force-free electrodynamics). More details on the geometric and effective action view point of FFE can be found in e.g. [20,21] and [22-25]. One should emphasise that the system of equations in eq. (1.4) is independent of the microscopic details of the cold plasma, which then strongly resembles hydrodynamic descriptions. In fact, it turns out that (1.4) can arise in a special limit where $T \ll \sqrt{|\mathbf{B}|}$ of a hydrodynamics description with one-form $\mathrm{U}(1)$ symmetry in $(1.3)$, see $[6,24,25] .{ }^{5}$

The existence of FFE is usually justified by saying that the cold plasma is, on one hand, dense enough to screen the electric field (1.4a) but, on the other hand, dilute enough so that force-free condition (1.4b) is applicable. This statement can be made more precise in the light of relations between the equations of FFE and hydrodynamics. Thus, we propose a criterion for testing the validity of FFE using the lifetime of non-conserved operators FFE, or equivalently, hydrodynamic description of cold plasma in the $T \ll \sqrt{|\mathbf{B}|}$ limit, is valid when the lifetime of all non-conserved operators is parametrically shorter than the time scale of interest. A key advantage of this approach is that the operator lifetime can be, in principle, computed explicitly from microscopic description and therefore allows one to find the 'cutoff' scale where FFE description should break down.

Computing the operator lifetime from microscopic description is, however, not always an easy task. In fact, we are not aware of a genuine computation directly from quantum electrodynamics (in the sense of [14]) when both $T$ and $\mathbf{B}$ are turned on. To simplify the computations, we shall demonstrate the validity of FFE in the strongly interacting magnetised plasma with a holographic dual as proposed in [27, 28] where the one-form U(1) global symmetry is taken into account via a two-form gauge field in the gravity dual. This provides two key advantages. First, the computation of correlation functions boils down to solving simple linearised differential equations (see e.g. [29]). Second, there is strong evidences that charge neutral operators, apart from energy and momentum, have a parametrically short lifetime in this class of theories. ${ }^{6}$ Therefore, we shall focus on non-conserved operators in

\footnotetext{
${ }^{5}$ Recasting of force-free electrodynamics in the hydrodynamic language also allows the systematic gradient expansions $[24,26]$. This could serve to classify correction to FFE in order to account for phenomena such as pulsar radio emission where $\mathbf{E} \cdot \mathbf{B} \neq 0$.

${ }^{6}$ To be more precise, it has been shown in $\mathcal{N}=4$ supersymmetric Yang-Mills theory, which constitutes the matter sector of the holographic model [27, 28], that there is no long-lived mode besides hydrodynamic modes at any $T \neq 0$ and $|\mathbf{B}|=0$ [30]. A similar conclusion was reached for the same theory in the charge neutral sector at finite non-dynamical magnetic field [31, 32].
} 
the electromagnetic sector of the theory: the electric flux operators, whose lifetime can be extracted via two-point correlation function as in (1.1). This will provide strong evidence for the validity of FFE limit in a strongly interacting holographic plasma.

On the technical side, the computations presented in this note show that there are no quasinormal modes present in the vicinity of the hydrodynamic regime $\omega / T \ll 1$ (and $\omega / \sqrt{|\mathbf{B}|} \ll 1)$. The pole in the electric flux correlation function in this regime then implies that the operator has a parametrically long lifetime which could interfere with the hydrodynamic modes. The presence of such long-lived mode can be determined analytically in the usual hydrodynamic regime of $\omega / T \ll 1$ for a large class of theories. It is usually difficult to go beyond this regime towards the limit $\omega / T \sim 1, \omega / \sqrt{|\mathbf{B}|} \ll 1$. Such computation can, however, be done analytically in the simple model of [27] thanks to the presence of the $\mathrm{BTZ} \times \mathbb{R}^{2}$ bulk geometry in the deep IR [33]. We should also note that the treatment of a (long-lived) non-hydrodynamic modes has been extensively used to determine the breakdown of hydrodynamic descriptions in the context of QFTs with holographic duals, see e.g. [34-37].

The remainder of this paper is organised as the follows. In section 2, we summarise the procedure involved in the computation of the two-point correlation function in the holographic dual to one-form global symmetry. In section 2.1, we outline the method for exploring the existence of decaying modes in the vicinity of the usual hydrodynamic limit $\omega / T \ll 1$ at $T / \sqrt{\mathbf{B} \mid} \ll 1$. Due to the simplicity of the bulk geometry, we are able to further extend the analysis to arbitrary value of $\omega / T$ with $\omega / \sqrt{|\mathbf{B}|} \ll 1$ and $T / \sqrt{|\mathbf{B}|} \ll 1$. This is described in section 2.2. Further open questions and future directions are discussed in section 3 .

\section{The holographic model}

A simple holographic dual to a strongly interacting field theory of matter charged under dynamical U(1) electromagnetism (that is, the dynamical plasma described by low energy MHD) and formulated in the language of higher-form symmetry was constructed in [27, 28]. We present a brief review here for completeness. The five-dimensional bulk theory is comprised of Einstein gravity coupled to a two-form bulk gauge field, $B_{\mu \nu}$, and a negative cosmological constant,

$S=\int d^{5} X \sqrt{-G}\left(R-2 \Lambda-\frac{L^{2}}{3} H_{a b c} H^{a b c}\right)+S_{\mathrm{bnd}}-\frac{1}{\kappa(\Lambda)} \int_{r=\Lambda} d^{4} x \sqrt{-\gamma}\left(n^{a} H_{a \mu \nu}\right)\left(n_{b} H^{b \mu \nu}\right)$,

where $H=d B$ and $B_{a b}$ is the bulk 2 -form gauge field, $\Lambda$ is the UV-cutoff, $n^{a}$ is the unit normal to the boundary, and $S_{\text {bnd }}$ denotes the Gibbons-Hawking and gravitational counter term. Roughly speaking, the two bulk fields $G_{a b}$ and $B_{a b}$, asymptote to $g_{\mu \nu}$ and $b_{\mu \nu}$ respectively, which then source the currents, $T^{\mu \nu}$ and $J^{\mu \nu}$.

$$
\left\langle T_{\mu \nu}\right\rangle \equiv \frac{2}{\sqrt{-g}} \frac{\delta S}{\delta g_{\mu \nu}}, \quad\left\langle J_{\mu \nu}\right\rangle \equiv \frac{1}{\sqrt{-g}} \frac{\delta S}{\delta b_{\mu \nu}}
$$


The generating functional takes the form,

$$
Z\left[g_{\mu \nu}, b_{\mu \nu}\right]=\left\langle\exp \left[i \int d^{4} x \sqrt{-g}\left(\frac{1}{2} T^{\mu \nu} g_{\mu \nu}+J^{\mu \nu} b_{\mu \nu}\right)\right]\right\rangle
$$

and diffeomorphism invariance and gauge symmetry lead to the following equations,

$$
\nabla_{\mu}\left\langle T^{\mu \nu}\right\rangle=(d b)_{\rho \sigma}^{\nu}\left\langle J^{\rho \sigma}\right\rangle, \quad \nabla_{\mu}\left\langle J^{\mu \nu}\right\rangle=0
$$

$H=d b$ is the three-form field strength of the two-form external source. The equilibrium solution of this holographic model is a domain wall interpolating between an asymptotic $A d S_{5}$ geometry in the UV ( $r \rightarrow \infty$ in our convention), and $B T Z \times \mathbb{R}^{2}$ in the near-horizon IR $\left(r=r_{h}\right)$. It is described by the following metric and gauge field

$$
\begin{aligned}
d s^{2} & =G_{a b} d X^{a} d X^{b}=-r^{2} f(r) d t^{2}+\frac{d r^{2}}{r^{2} f(r)}+e^{2 V(r)}\left(d x^{2}+d y^{2}\right)+e^{2 W(r)} d z^{2}, \\
B & =h(r) d t \wedge d z \quad \text { with } \quad \star_{5} H=\mathcal{B} d x \wedge d y
\end{aligned}
$$

Modulo the subtleties due to the mixed boundary conditions, this is nothing but the hodge dual of the magnetised black brane solution of [33]. The radial coordinate is chosen such that $r \rightarrow \infty$ corresponds to the usual asymptotic $A d S_{5}$ with

$$
f(r)=1, \quad e^{2 V(r)}=e^{2 W(r)}=r^{2}
$$

in the $r \rightarrow \infty$ limit. The $B T Z \times \mathbb{R}^{2}$ solution near the horizon can be written as

$$
f(r)=3\left(1-\frac{r_{h}^{2}}{r^{2}}\right), \quad e^{2 V}=\frac{\mathcal{B}}{\sqrt{3}}, \quad e^{2 W}=3 r^{2} .
$$

The temperature is set by the horizon radius via $4 \pi T=r_{h}^{2}\left|f^{\prime}\left(r_{h}\right)\right|=6 r_{h} / L^{2}$. We set $L=1$ for simplicity. Note also that $\mathcal{B}$ is related to the $z$-component of the 'physical' magnetic field $\mathbf{B}$ which differs by a prefactor $L$ or the 2 -form gauge field coupling in the bulk (e.g. if one were to define the action with $\left.S \sim \int\left(1 / g^{2}\right) H^{2}\right)$. We will keep using $\mathcal{B}$ to emphasise its holographic origin but there is no harm in thinking of it as simply $\mathbf{B}$.

One interesting feature of this model is that the leading divergence of $B_{\mu \nu}$ in the Fefferman-Graham expansion is logarithmic. Thus, the definition of the source $b_{\mu \nu}$ requires mixed boundary condition

$$
b_{\mu \nu}=B_{\mu \nu}(\Lambda)-\frac{1}{\kappa(\Lambda)}\left\langle J_{\mu \nu}\right\rangle, \quad \text { with } \quad\left\langle J^{\mu \nu}\right\rangle=-\sqrt{-G} n_{\alpha} H^{\alpha \mu \nu}
$$

Requiring the source $b_{\mu \nu}$ to be independent of the UV cutoff fixes the form of the 'coupling constant' $1 / \kappa(\Lambda)$ which turns out to be logarithmically running. This is a common feature for fields with this type of near-boundary behaviour where the counterterm also plays the role of the double-trace deformation [38, 39], see also [27, 28] for a discussion in the present context. Mapping $J^{\mu \nu}$ in to a more familiar dynamical field strength via $J^{\mu \nu}=\frac{1}{2} \epsilon^{\mu \nu \rho \sigma} F_{\rho \sigma}$, one can see that the double-trace deformation plays a role similar to the Maxwell term for the dynamical gauge field in the dual QFT with $1 / \kappa(\Lambda)$ as a (logarithmically running) electromagnetic coupling. 
The finite part of $1 / \kappa(\Lambda)$ plays a crucial role in this setup. While the finite counterterm in the ordinary bulk Maxwell theory simply results in a contact term in the correlation function, the mixed boundary condition for $B_{a b}$ implies the existence of the purely decaying mode $\omega=-i / \tau_{E}$ that can interfere with the gapless hydrodynamic excitation. This is nothing but the life-time of the electric flux operator $Q_{E} \sim \int d S_{i j} J^{i j}$ which appears in the following correlation function $[28,34]$

$$
\left\langle J^{i j}(t) J^{k l}(0)\right\rangle \sim \exp \left(-i t / \tau_{E}\right) .
$$

Note that, due to the anisotropy introduced by finite equilibrium magnetic field, the value of $\tau_{E}$ depends on which direction of the electric field in consideration. The limit where $\tau_{E}$ is small, but finite, compared to the length scale of interest (set by temperature or magnetic flux density) is of particular interest as it allows one to extract $\tau_{E}$ analytically, via a matching procedure that we outline below. As argued in the introduction, the lifetime of the electric flux determines the validity of MHD and FFE description.

\subsection{Linearised solutions in $\omega / T \ll 1$ limit and matching procedure}

In this section, we outline the computation required to obtain the relaxation time of the electric field. We focus on the hydrodynamic regime where $\omega / T \ll 1$, and the low temperature limit $^{7} T / \sqrt{|\mathbf{B}|} \ll 1$. This allows us to solve the bulk equation of motion analytically via a matching method similar to that was employed in [30] (see also [34] for a recent review). We consider the decay rate of the electric field both along and perpendicular to the equilibrium magnetic field denoted by $E^{\|}=J^{x y}$ and $E^{\perp}=J^{x z}, J^{y z}$ respectively.

Before proceeding, let us summarise the matching procedure for the $\omega / T \ll 1$ expansion. It involves separating the bulk into three suitably defined pieces: inner region, intermediate region and outer region. The inner region is a suitably defined region close to the horizon while the outer region is defined to be the range of $r$ such that $\omega / r \ll 1$ so that one can drop terms quadratic in $(\omega / r)^{2}$, which includes the near boundary region. The integration constants of the solution in the outer region are determined by matching the form of inner region solution for intermediate value of $r$ that connect the two regions together. In our case, this is the region of $r$ close to $r_{h}$ but

$$
\frac{\omega}{T} \log f(r) \ll 1
$$

This intermediate region defined above is also consistent with the outer region assumption where $\omega / r \ll 1$ and thus we are able to match the two solution together. Note that, while this procedure is applicable to any bulk solution with event horizon, the limit $\omega / T \ll 1$ is crucial.

We now present the key equations and resulting lifetime of the electric flux.

\subsubsection{Perturbation parallel to equilibrium magnetic field}

As the magnetic field in equilibrium points along the $z$-direction, we are interested in $E^{\|}=\frac{1}{2} \varepsilon^{z x y}\left\langle J_{x y}\right\rangle$. The corresponding bulk perturbation is $\delta B_{x y}$ which decouples from the

\footnotetext{
${ }^{7}$ Similar computation for the holographic theory dual to a system with ordinary(zero-form) U(1) symmetry can be found in e.g. [40, 41].
} 
metric perturbation in the zero wave vector limit. The bulk equation of motion can be written as

$$
\left(r^{2} f e^{W-2 V} \delta B_{x y}^{\prime}\right)^{\prime}+\frac{\omega^{2}}{r^{2} f} e^{W-2 V} \delta B_{x y}=0
$$

where $(\ldots)^{\prime}$ denotes a derivative w.r.t. the radial coordinate $r$. The inner region solution for $\delta B_{x y}$, where we substitute the $B T Z \times \mathbb{R}^{2}$ solution for $f, V, W$, with the ingoing boundary condition can be written as

$$
\delta B_{x y}^{\text {inner }}=c^{H} \exp \left(-\frac{i \omega}{4 \pi T} \log f(r)\right)
$$

The outer region solution can be obtained by considering the solution at linear order in $\omega / r$ and one obtains,

$$
\begin{aligned}
\delta B_{x y}^{\text {outer }}(r) & =c_{1}-c_{2}\left(\log \Lambda-\int_{\mathrm{r}=r}^{\Lambda} d \mathrm{r} \frac{e^{2 V(\mathrm{r})-W(\mathrm{r})}}{\mathrm{r}^{2} f(\mathrm{r})}\right) \\
& =c_{1}-c_{2}\left(\log r-\phi(r)+\left.\frac{e^{2 V-W}}{r_{h}^{2} f^{\prime}}\right|_{r=r_{h}} \log f\right),
\end{aligned}
$$

where $\phi(r)$ is a function regular everywhere in the bulk defined as

$$
\phi(r)=\int_{\mathrm{r}=r}^{\Lambda} d \mathrm{r}\left[\frac{e^{2 V(\mathrm{r})-W(\mathrm{r})}}{\mathrm{r}^{2} f(\mathrm{r})}-\left(\frac{e^{2 V(\mathrm{r})-W(\mathrm{r})}}{r_{h}^{2} f^{\prime}(\mathrm{r})}\right)_{\mathrm{r}=r_{h}} \frac{f^{\prime}(\mathrm{r})}{f(\mathrm{r})}-\frac{1}{\mathrm{r}}\right] .
$$

This parametrisation allows us to single out leading contributions that dominate when considering the solution near $r=\Lambda$, where $\phi(r)$ and $\log \left(e^{-2 V} r^{2} f\right)$ vanish, as well as near $r \approx r_{h}$ where the $\log f$ term dominates. The integration constants $c_{1}, c_{2}$ in (2.13) are related to the source $b_{\mu \nu}$ and the 2 -form current $\left\langle J^{x y}\right\rangle$. The precise relations can be obtained via eq. (2.8) to be

$$
\left\langle J^{x y}\right\rangle=c_{2}, \quad b_{x y}=c_{1}-\left(\log \Lambda+\frac{1}{\kappa(\Lambda)}\right) c_{2}
$$

Note that, for the source to be independent of the UV cutoff, one requires $\kappa(\Lambda)^{-1}=$ finite term $-\log \Lambda$. This is the logarithmically running coupling usually found in a doubletrace deformed theory and resembles the running of electromagnetic coupling as pointed out in $[27,28,34]$.

For the outer and inner region solutions to match, we consider both solutions in the intermediate region where we can write the inner solution as

$$
\exp \left(-\frac{i \omega}{4 \pi T} \log f\right) \approx 1-i \frac{\omega}{4 \pi T} \log f+\mathcal{O}\left(\frac{\omega}{T}\right)^{2}
$$

The matching condition $\delta B_{x y}^{\text {inner }}=\delta B_{x y}^{\text {outer }}$ in this region prompts yield the following algebraic relations between the boundary quantities $b_{x y},\left\langle J_{x y}\right\rangle$ :

$$
\begin{aligned}
\frac{i \omega}{4 \pi T} c^{H} & =\left(\frac{\mathcal{B} / r_{h}}{3 r_{h}^{2} f^{\prime}\left(r_{h}\right)}\right)\left\langle J_{x y}\right\rangle \\
c^{H} & =b_{x y}+\left[\frac{1}{\kappa(\Lambda)}+\log \left(\frac{\Lambda}{r_{h}}\right)+\phi\left(r_{h}\right)\right]\left\langle J_{x y}\right\rangle .
\end{aligned}
$$




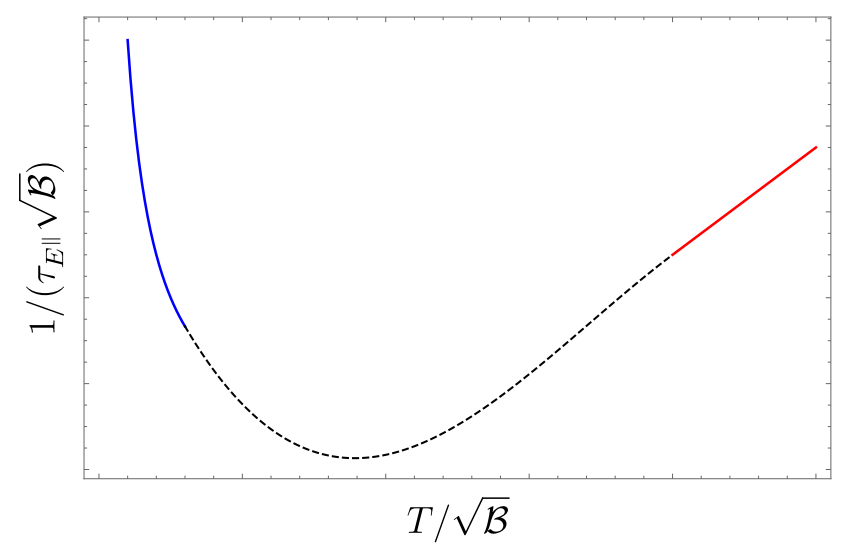

Figure 2. A sketch of the decay rate (inverse of the lifetime) of the electric field as a function of $T / \sqrt{\mathcal{B}}$, measured in the unit of $\sqrt{\mathcal{B}}$. The high temperature regime (red) depict the result of decay rate at zero magnetic field found in $[28,34]$ which has the same temperature dependence as in (1.1)-(1.2). In the low temperature regime (blue), however, the operator lifetime becomes those found in (2.17).

Solving these equations at vanishing source $b_{x y}=0$ yields the spectrum of the form $\omega=-i / \tau_{E \|}$ where $\tau_{E \|}$ is the lifetime of the electric flux parallel to the equilibrium magnetic field. This is the first key result that we advertised earlier, namely

$$
\tau_{E \|}=\frac{2 \pi T}{\mathcal{B}}\left(e_{r}^{-2}+\phi\left(r_{h}\right)\right)
$$

where we write $e_{r}^{-2}=\log \left(\Lambda / r_{h}\right)+\kappa(\Lambda)^{-1}$ which plays the role of renormalised electromagnetic coupling. More details on the $T / \sqrt{\mathcal{B}}$ dependence of $\phi\left(r_{h}\right)$ can be found in appendix A.

Let us discussed a formal limit similar to [34] in order to make sense of this result that is consistent with the assumption $\omega / T \ll 1$. While the integral $\phi\left(r_{h}\right)$ can be a dimensionless function of $T$ and $\mathcal{B}$, the renormalised electromagnetic coupling can be chosen in such a way that $e_{r}^{-2} \gg \phi\left(r_{h}\right)$ and $e_{r}^{-2} T^{2} / \mathcal{B} \ll 1$ so that $\omega \tau_{E \|} \sim \omega / T \ll 1$. The second limit is essential as the matching procedure assumes that $\omega / T \ll 1$ and the solution outside this regime has to be discarded. Taking these factors into account, one concludes that the temperature dependence of the electric flux is different from the high temperature $T / \sqrt{\mathcal{B}} \gg 1$ limit where $\tau_{E} \sim 1 / T$ (see figure 2). Naively taking the limit $T \rightarrow 0$ in (2.17) will result in the vanishing lifetime of the electric flux in contrast to the result in (1.2). However, one has to carefully remove the limit $\omega / T \ll 1$ in order to access the lower temperature limit $\omega / T \sim 1, \omega / \sqrt{\mathcal{B}} \ll 1$ as explained in section 2.2 .

\subsubsection{Perturbation perpendicular to equilibrium magnetic field}

Unlike the previous case, the perturbation $\delta B_{x z}$ that corresponds to $E^{\perp}=\frac{1}{2} \epsilon^{y z x}\left\langle J_{z x}\right\rangle$ is coupled to the metric perturbation. This is manifest in the equations of motion

$$
\begin{aligned}
\frac{d}{d r}\left(r^{2} f e^{-W} \delta B_{x z}^{\prime}+\mathcal{B}\left(\delta G_{t}^{x}\right)\right)+\frac{\omega^{2} e^{-W}}{r^{2} f} \delta B_{x z} & =0, \\
\frac{d}{d r}\left(e^{4 V+W}\left(\delta G_{t}^{x}\right)^{\prime}+4 \mathcal{B} \delta B_{x z}\right) & =0,
\end{aligned}
$$


where $\delta G_{\mu \nu}$ denotes the metric perturbations. Note that the coupled perturbations $\left\{\delta B_{x z}, \delta G_{t x}\right\}$ and $\left\{B_{y z}, \delta G_{y z}\right\}$ are equivalent due to $\mathrm{SO}(2)$ symmetry in the plane perpendicular to the equilibrium magnetic field. Also, the second equation of motion in (2.18) can be written in a total derivative form $d \pi_{t x} / d r=0$ with $\pi_{t x}$ is related to the momentum $\left\langle T^{t x}\right\rangle$. Since we are working in the zero wavevector limit, the conservation of momentum implies that $\pi_{t x}=0$ in Fourier space (which can be shown explicitly using the $r x$-component of the Einstein equation).

The solution for $\delta B_{x z}, \delta G_{t x}$ in the outer region can be found by using the property of the background geometry combined with the Wronskian method as in [34]. To be more precise, one first notes that the time-independent solution of the magnetised black brane can be written in a total derivative form, which implies the existence of two radially conserved currents.

$$
\begin{aligned}
& Q_{1}=r^{2} f\left(V^{\prime}-W^{\prime}\right) e^{2 V+W}+2 \mathcal{B} h(r)=0, \\
& Q_{2}=e^{4 V+W} \frac{d}{d r}\left(e^{-2 V} r^{2} f\right)-4 \mathcal{B} h(r)=s T,
\end{aligned}
$$

where we write the equilibrium ansatz for the gauge field as $B=h(r) d t \wedge d z$ with gauge choice $h\left(r_{h}\right)=0$, which, together with the horizon regularity, sets $Q_{1}=0$. The relation between $h(r)$ and the 3 -form field strength is

$$
e^{2 V-W} h^{\prime}=\mathcal{B} .
$$

More details on obtaining these radially conserved quantities can be found in e.g. [42]. With this ansatz, we can compare (2.18) and (2.19) and find that one of the solutions of (2.18) when $\omega / r \rightarrow 0$ are

$$
\delta B_{x z}=\Phi_{1}(r)=h(r)+\frac{s T}{4 \mathcal{B}}, \quad \delta G_{t}^{x}=\Psi_{1}(r)=-e^{-2 V} r^{2} f .
$$

One can use the Wronskian method to find a pair of solution of (2.18) that are linearly independent to $\left\{\Phi_{1}, \Psi_{1}\right\}$. These solutions are

$$
\Phi_{2}(r)=\frac{1}{4 \mathcal{B}}-\int_{r}^{\infty} d \mathrm{r}\left(\frac{\mathcal{B} e^{W(\mathrm{r})} \Psi_{2}(\mathrm{r})}{\mathrm{r}^{2} f(\mathrm{r})}\right), \quad \Psi_{2}(r)=\Psi_{1}(r) \int_{r}^{\infty} d \mathrm{r}\left(\frac{e^{-W(\mathrm{r})}}{\mathrm{r}^{4} f(\mathrm{r})^{2}}\right)
$$

As a result, the outer region solution can be written as

$$
\left(\begin{array}{c}
\delta B_{x z}^{\text {outer }} \\
\left(\delta G_{t}^{x}\right)^{\text {outer }}-\frac{1}{\mathcal{B}} \mathcal{J}_{x z}
\end{array}\right)=c_{1}\left(\begin{array}{l}
\Phi_{1} \\
\Psi_{1}
\end{array}\right)+c_{2}\left(\begin{array}{l}
\Phi_{2} \\
\Psi_{2}
\end{array}\right)
$$

where $\mathcal{J}_{x z}:=\left(r^{2} f e^{-W} \delta B_{x z}^{\prime}+\mathcal{B} \delta G_{x}^{t}\right)$ is an integration constant of (2.18) at $\omega=0$. One can substitute the $B T Z \times \mathbb{R}^{2}$ ansatz into the solution in (2.22) to check that $\Phi_{1}, \Psi_{1,2}$ are finite at $r=r_{h}$ while $\Phi_{2}$ is singular. It is convenient to separate out the singular part of $\Phi_{2}$ in the following form

$$
\Phi_{2}(r)=\phi_{2}(r)-\left(\frac{\mathcal{B} e^{W} \Psi_{2}}{r^{2} f^{\prime}}\right)_{r=r_{h}} \log f(r)
$$


where $\phi_{2}(r)$ is the integral in (2.21) with the logarithmic divergence subtracted. The boundary condition where the source for both metric and 2-form gauge field fluctuation vanishes corresponds to the following values of $c_{1}$ and $c_{2}$

$$
c_{1}=\frac{\mathcal{J}_{x z}}{\mathcal{B}}, \quad c_{2}=-4\left(\frac{s T}{4 \mathcal{B}}+h(\Lambda)+\frac{\mathcal{B}}{\hat{\kappa}(\Lambda)}\right) \mathcal{J}_{x z}
$$

One can also check that $\mathcal{J}_{x z}$ is identical to the one-point function $\left\langle\delta J^{x z}\right\rangle$ via the definition (2.8). Note also that the ratio $c_{2} / \mathcal{J}_{x z}$ is finite due to the cancellation of the logarithmic divergence of $1 / \kappa(\Lambda)$ and that of the near boundary solution of $h(r)$, obtained via (2.19c).

Let us also pointed out another way to organise the equations of motion for $\delta B_{x z}$. It turns out that (2.18) can be combined into a single equation of motion that reduces to a total derivative form at $\omega=0$. Following the procedure in e.g. [43] and some manipulation, we find

$$
\left(\left[e^{4 V+W}\left(e^{-2 V} r^{2} f\right)^{\prime}\right]^{2} r^{2} f e^{-W} \delta \tilde{B}_{x z}^{\prime}\right)^{\prime}+\frac{\omega^{2}}{r^{2} f e^{W}}\left[e^{4 V+W}\left(e^{-2 V} r^{2} f\right)^{\prime}\right]^{2} \delta \tilde{B}_{x z}=0
$$

where $\delta \tilde{B}_{x z}=\delta B_{x z} /\left[e^{4 V+W}\left(e^{-2 V} r^{2} f\right)^{\prime}\right]$. The outer region solution of (2.25) is easily obtained and can be shown to be identical to those of (2.22).

We can now proceed to the inner region solution. This can be found by solving eq. (2.25) and one find

$$
\delta B_{x z}^{\text {inner }}=c^{H} \exp \left(-\frac{i \omega}{4 \pi T} \log f(r)\right) .
$$

In the intermediate region, we apply the expansion in (2.15). The coefficients $c_{1}, c_{2}$ are related to $c^{H}$ via

$$
\begin{aligned}
\left(-\frac{i \omega}{4 \pi T}\right) c^{H} & =-\left(\frac{\mathcal{B} e^{W} \Psi_{2}}{r^{2} f^{\prime}}\right)_{r=r_{h}} c_{2}, \\
c^{H} & =\left(\frac{s T}{4 \mathcal{B}}\right) c_{1}+\phi_{2}\left(r_{h}\right) c_{2},
\end{aligned}
$$

Substituting the form of $c_{1}, c_{2}$ in terms of $\left\langle\delta J_{x z}\right\rangle$, we can write the relations in a form similar to $\left\langle\delta J_{x y}\right\rangle$, namely

$$
\left(-i \omega+\frac{1}{\tau_{E^{\perp}}}\right)\left\langle\delta J_{x z}\right\rangle=0 .
$$

In the case of vanishing sources, we can write $\frac{c_{2}}{c_{1}}=-4 \mathcal{B}\left(\frac{s T}{4 \mathcal{B}}+h(\Lambda)+\frac{\mathcal{B}}{\kappa(\Lambda)}\right)$ and the relaxation time of the electric field perpendicular to the equilibrium magnetic field is

$$
\tau_{E^{\perp}}=\frac{\sqrt{3}}{2 \pi T \mathcal{B} \Psi_{2}\left(r_{h}\right)}\left[\frac{s T}{4 \mathcal{B}} \frac{c_{1}}{c_{2}}+\phi_{2}\left(r_{h}\right)\right]
$$

In contrast to the result at $e_{r}^{-2} \gg 1$ and zero equilibrium magnetic field in $[28,34]$, the lifetime at strong magnetic field $\mathcal{B} / T^{2}$ has a very different form. To see this, it is useful to examined that the combinations that enter the lifetime as follows

$$
\Psi_{2}\left(r_{h}\right) \propto \frac{1}{\mathcal{B} T^{2}}, \quad \phi_{2}\left(r_{h}\right) \propto \frac{1}{\mathcal{B}}, \quad \frac{c_{1}}{c_{2}} \propto \frac{1}{\mathcal{B}^{2}} \quad \text { for large } 1 / \kappa(\Lambda)
$$


with proportionality constants given by some numbers of order $\mathcal{O}(1)$. In the limit of large electromagnetic coupling $1 / \kappa(\Lambda) \gg 1$ and $\mathcal{B} / T^{2} \gg 1$, we find that this gives a short lifetime of the form $\tau_{E^{\perp}} \propto T / \mathcal{B}$. However, the location of this decaying mode $\omega=-i / \tau_{E^{\perp}}$ lies outside the hydrodynamic regime $\omega / T \ll 1$. Thus, one conclude that there are no modes with long lifetime in this regime. ${ }^{8}$

\subsection{Checking $T \gtrsim 0$ limit in $\omega / \sqrt{|\mathrm{B}|} \ll 1$ regime}

While the result in the previous section strongly indicated that the electric flux lifetime becomes very short at extremely low temperature, the simplicity of the holographic model also allows us to extend the analysis beyond the usual hydrodynamic $\omega / T \ll 1$ regime. We will first show that the zero temperature theory does not support the purely decaying mode of the form $\omega=-i / \tau$ in the small $\omega / \sqrt{|\mathbf{B}|}$ regime. Next, we further extend the regime of validity to that of $\omega / \sqrt{|\mathbf{B}|} \ll 1$ but for arbitrary $\omega / T$. The purpose of the latter is to show that $\tau_{E} \propto T / \sqrt{|\mathbf{B}|}$ without relying on the $\omega / T \ll 1$ limit.

\subsubsection{Zero temperature}

A simple argument for the non-existence of such a slowly decaying mode, is the presence of Lorentz symmetry at zero temperature on the $A d S_{3}$ submanifold in the deep infrared. On the other hand, one can also show this, using matching methods similar to those in $[40,45,46]$.

To obtain this result, one first realises that the geometry of the magnetised black brane is that of an interpolation between IR $A d S_{3} \times \mathbb{R}^{2}$ and UV $A d S_{5}$. Roughly speaking, the IR geometry starts to becomes a good approximation as one starts to probe the scale below the magnetic field i.e. $r \sim \sqrt{|\mathbf{B}|}$. The inner and outer regions are defined such that they start off from the IR and UV geometry respectively, and extend to cover the overlap region (see figure 3). This is achievable when $\omega / \sqrt{|\mathbf{B}|} \ll 1$.

For concreteness, let us demonstrate how this works in the $E^{\|}$channel that involves the bulk field $\delta B_{x y}$ governed by eq. (2.11). The solution can be written in the same form as (2.13) evaluated at zero temperature (i.e. $r_{h}=0$ ). It is worth noting that the singular behaviour near $r / \sqrt{\mathcal{B}} \rightarrow 0$ is different from that in earlier section. Instead, it can be written as

$$
\delta B_{x y}^{\text {outer }}(r)=c_{1}-c_{2}\left(\log \Lambda-\bar{\phi}(r)+\frac{\mathcal{B} / 3}{6 r^{2}}\right)+\mathcal{O}\left(\frac{\omega^{2}}{r^{2}}, \frac{\omega^{2}}{r^{2}} \log \left(\frac{\omega}{r}\right)\right)
$$

where the integration constants can be related to source and response via (2.8). It is worth noting that the logarithmic divergence appears at order $\omega^{2}$. This is can be confirmed via Frobenius analysis in $A d S_{5}$ region (see e.g. [47]) and $A d S_{3} \times \mathbb{R}^{2}$ region (see appendix B). The prefactor of the $r^{-2}$ divergence is obtained by evaluating $e^{2 V(r)-W(r)} / f(r)$ at the horizon $r \rightarrow 0$. Here $\bar{\phi}(r)$ is the integral in (2.13) subtracted by the $r^{-2}$ divergent and logarithmic

\footnotetext{
${ }^{8}$ Note also that, if one were to perform this analysis for a perturbation in the holographic dual to a theory with zero-form $\mathrm{U}(1)$ at $T>0, \mu=0$ (as in [30], see also [34]), one would find a spectrum of the form $\omega \sim T$. This solution is spurious as it lies outside the hydrodynamic regime $\omega / T \ll 1$ and, in fact, is not present in the genuine spectrum obtained numerically at finite $\omega / T$ [44].
} 


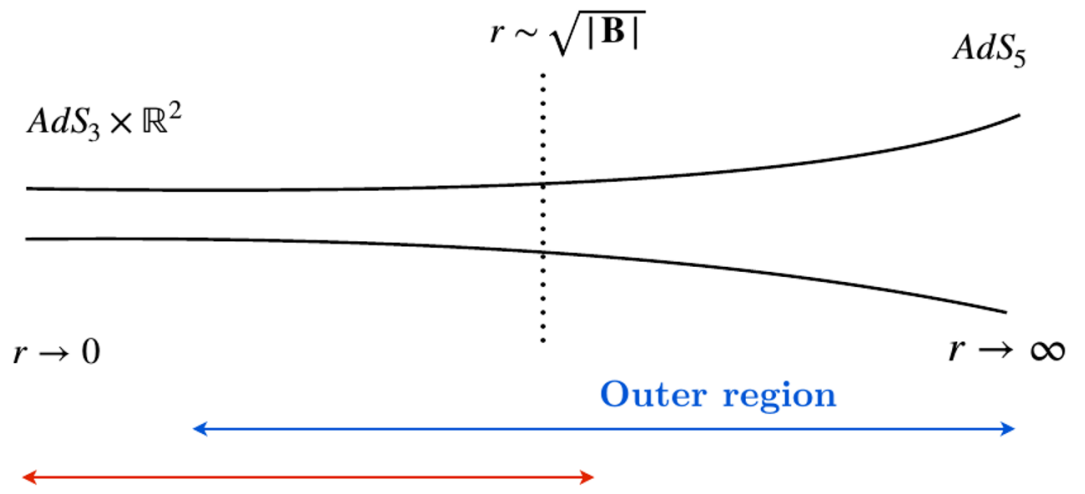

Inner region

Figure 3. A sketch of the bulk geometry at zero temperature. The inner region, whose solutions only depends on the ratio $\omega / r$ extended from the near horizon limit $r \rightarrow 0$ to the one where $\omega / r \sim \omega / \sqrt{|\mathbf{B}|} \rightarrow 0$ as we are working in the $\omega / \sqrt{|\mathbf{B}|} \ll 1$ limit. The outer region is defined to be the region where the $\omega^{2} / r^{2}$ and higher power in $\omega / r$ is suppressed, which can be extended toward $r \gg \sqrt{|\mathbf{B}|}$ as long as the frequency is small.

divergent pieces. The resulting integral evalutated from $r=r_{0} \sim \sqrt{\mathcal{B}}$ of the overlapping region to the UV cutoff $r=\Lambda$ is finite and its number is not extremely relevant for us as long as one keep $e_{r}^{-2}$ large.

Next, we consider the inner region solution, which can be obtained by solving (2.11) in the $A d S_{3} \times \mathbb{R}^{2}$ region. Upon imposing horizon regularity at $r \rightarrow 0$, we find that the inner region solution is

$$
\delta B_{x y}^{\text {inner }}=c^{H} \zeta K_{1}(\zeta), \quad \zeta=\frac{3 \omega}{r}
$$

For these two branches of solutions to match, we extend the inner region solution to the regime where $\zeta=\omega / r \ll 1$. We find that the 'near boundary' expansion takes the form

$$
\delta B_{x y}^{\text {inner }}=c^{H}\left(1+\frac{1}{2} \gamma \zeta^{2}+\frac{1}{2} \zeta^{2} \log \zeta+\ldots\right)
$$

Matching this solution to the outer region, we find that $c_{2} \propto \omega^{2}$ unlike what happened in the previous section. Carrying on the matching procedure, we find that the polynomial governing the spectrum only depends on $\omega^{2}$ and thus rules out the purely imaginary mode $\omega=-i / \tau$. The same argument can also be made for the $E^{\perp}$ channel involving $\delta B_{x z}$. This is because, the part that is relevant to the matching procedure only depends on $\zeta^{2}$. See appendix B for more details on the form of $\delta B_{x z}$ in the $A d S_{3} \times \mathbb{R}^{2}$ region.

\subsection{2 $T \lesssim \omega \ll \sqrt{|\mathrm{B}|}$ limit}

In this section, we show that the electric flux lifetime can also be obtained regime where $\omega / T \gtrsim 1$ and $\omega / \sqrt{|\mathbf{B}|} \ll 1$ while keeping $\sqrt{|\mathbf{B}|} / T \gg 1$. The calculations closely resembles that of the zero temperature case except that the deep IR geometry is now $B T Z \times \mathbb{R}^{2}$ instead of $A d S_{3} \times \mathbb{R}^{2}$. Figure 4 illustrates this geometry where the $A d S_{5}$ joined with the $B T Z \times \mathbb{R}^{2}$ at the 'boundary' $A d S_{3} \times \mathbb{R}^{2}$ of the IR geometry. We will only focus on the 


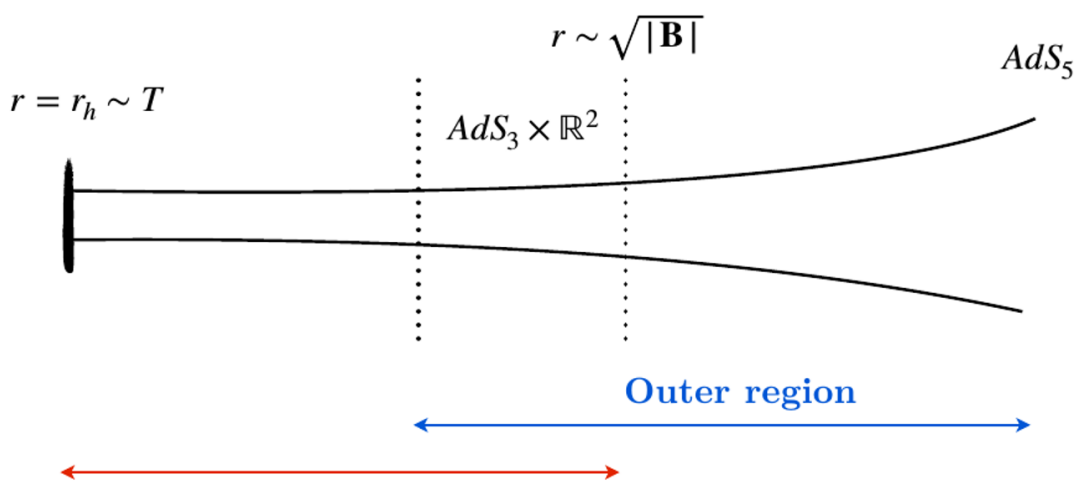

Inner region $B T Z \times \mathbb{R}^{2}$

Figure 4. A sketch of the bulk geometry at low temperature $T \ll \sqrt{|\mathbf{B}|}$. The inner region, whose solutions only depends on the ratio $\omega / r$ extends from the near horizon limit $r \rightarrow r_{h} \ll \sqrt{\mathbf{B}}$ to the one where $\omega / r \sim \omega / \sqrt{|\mathbf{B}|} \ll 1$, which corresponds to the near boundary region of $B T Z \times \mathbb{R}^{2}$ geometry, described by $A d S_{3} \times \mathbb{R}^{2}$. The outer region is defined to be the region where $\omega^{2} / r^{2}$ (and higher powers) is negligible and, therefore, can be extended toward $r \sim \sqrt{|\mathbf{B}|}$ in the $\omega / \sqrt{|\mathbf{B}|} \ll 1$ limit.

$E^{\|}$fluctuations as it is the only channel that contains the decaying modes in the $\omega / T \ll 1$ regime. Similar computation for this type of geometry can also be found in [46].

The outer region solution, which extends from the UV $A d S_{5}$ to the intermediate $A d S_{3} \times \mathbb{R}^{2}$ region has the same form as in (2.31). This is possible only in the limit where $\sqrt{\mathcal{B}} \gg T$ so that $r / \sqrt{\mathcal{B}}$ is always much greater than $T / \sqrt{\mathcal{B}} \sim r_{h} / \sqrt{\mathcal{B}}$ in this region.

The inner region solution in the $B T Z \times \mathbb{R}^{2}$ region can be expressed in terms of a hypergeometric function (upon imposing ingoing boundary condition)

$$
\delta B_{x y}^{\text {inner }}=c^{H}\left(1-\frac{r_{h}^{2}}{r^{2}}\right)^{-i \mathfrak{w} / 2}{ }_{2} F_{1}\left(-\frac{i \mathfrak{w}}{2},-\frac{i \mathfrak{w}}{2},-\frac{i \mathfrak{w}}{2} ; 1-\mathfrak{w} ; 1-\frac{r_{h}^{2}}{r^{2}}\right)
$$

where $\mathfrak{w}=\omega /(2 \pi T)=\omega / 3 r_{h}$. Extending this solution in the $r \gg r_{h}$ limit (which is possible due to $r_{h} / r \rightarrow 0$ as we approach the limit $\omega / r \rightarrow 0$ ) yields the following expansion [48]

$$
\delta B_{x y}^{\text {inner }} \propto c^{H}\left[1+\frac{i \omega r_{h}}{6 r^{2}}+\frac{1}{4}\left(\frac{\omega}{3 r}\right)^{2}\left(2-2 \gamma-2 \psi(1-i \mathfrak{w} / 2)-\log \left(\frac{r_{h}^{2}}{r^{2}}\right)\right)+\mathcal{O}\left(\omega^{3}\right)\right]
$$

where $\psi(x)$ is the digamma function and the constants of proportionality are combinations of gamma functions that can be absorbed in the definition of $c^{H}$. The first two terms in [...] are what important for us. By working to leading order in $\omega / r \ll 1$ as one approaches the intermediate $A d S_{3} \times \mathbb{R}^{2}$ region, we find the following matching solution

$$
c_{1}-c_{2} \log (\Lambda / \sqrt{\mathcal{B}})+\bar{\phi}=c^{H}, \quad\left(\frac{\mathcal{B}}{3}\right) c_{2}=i \omega\left(\frac{2 \pi T}{3}\right) c^{H}
$$

We can convert $c_{1}$ to the source $b_{x y}$ and $c_{2}$ as done in the previos sections. Upon taking $e_{r}^{-2} \gg \bar{\phi}$ (so that the solution lies in the regime of validity $\omega / \sqrt{\mathcal{B}} \ll 1$ ), we find the solution of the form $\omega=-i / \tau_{E \|}$ where $\tau_{E \|}$ is the same as in (2.17). This indicates that the lifetime indeed grows as $T / \sqrt{\mathcal{B}}$ increases regardless of the ratio $\omega / T$. 


\section{Conclusion}

The higher-form symmetry viewpoint of magnetohydrodynamics and its low temperature incarnation, the force-free electrodynamics, leads to new insights. The central focus of the present work was to established the absence of long-lived non-conserved operators. In turn, this indicates the validity of a hydrodynamic description at low temperature and strong magnetic field. The question of whether the only operators that govern the deep IR dynamics are the conserved charges is important and ought to be asked before any quantitative attempt is made to study hydrodynamic properties (such as shear viscosity etc). All non-conserved operators must decay much faster than the scale of interest if a hydrodynamic interpretation is to be meaningful.

We work with a holographic model which shares the same global symmetry as that of the plasma, namely only the energy, momentum and magnetic flux commute with the Hamiltonian. The model is simple enough for the lifetime of electric flux to be determined by classical bulk dynamics and the precise question is whether or not the electric flux is sufficiently long-lived to interfere with hydrodynamic modes. Due to the anisotropy of the system in the presence of a strong expectation value of magnetic field, the lifetime of the electric field depends on its orientation. Our results can be summarised as follows

- For electric flux $E_{\|}$parallel to the magnetic field, the lifetime has a strong dependence on the double-trace coupling $\kappa$ which plays a role similar to the renormalised electromagnetic coupling. In the extreme limit of $e_{r}^{-2} \gg|\mathbf{B}| / \mathbf{T}^{\mathbf{2}}$, the lifetime can be large enough to be detectable by the analytic computation in both the 'usual' hydrodynamic regime $\omega / T \ll 1$ and on even lower temperature regime where $\omega / \sqrt{\mathbf{B}} \ll 1$ while $\omega / T$ may remains finite. We found that the lifetime becomes shorter as one decreases the ratio of $T / \sqrt{|\mathbf{B}|}$. The latter indicates that the lifetime will become extremely short in the extremely strong magnetic field regime $T / \sqrt{|\mathbf{B}|} \ll 1$ and cannot interfere with the low energy regime of $\omega / \sqrt{|\mathbf{B}|} \ll 1$ where the FFE limit is thought to be applicable.

- For the component of electric flux $E_{\perp}$ perpendicular to the magnetic field, we find that there is no pole in the vicinity of $\omega / T \ll 1$. The dependence of the lifetime on the renormalised electromagnetic coupling disappears as one approaches the strong magnetic field limit.

We also performed a consistency check at $T \rightarrow 0$ to ensure that there are no modes in the deep IR limit of $\omega / \sqrt{|\mathbf{B}|} \ll 1$. In this regime, the modes that indicate (potentially) long lifetime of $E_{\|}$disappear from the low energy spectrum as anticipated.

These computations are basic checks on the validity of FFE description. In the holographic context, it would be interesting to check if all the accessible non-conserved operator truly have a parametrically short lifetime as well as confirming the low energy spectrum predicted by force-free electrodynamics (and its subsequent derivative corrections). Extraction of FFE effective action from gravity akin to [49-51] or the full constitutive relation as in [52-54] would be desirable as a definitive proof of FFE description in the dynamically 
magnetised black brane geometry. Last but not least, it would be very interesting to investigate operators lifetime in (weakly coupled) quantum electrodynamics at finite $T$ and B to better understand FFE and its limitations in a system more directly connected to astrophysical plasma than the strongly coupled holographic model considered here.

\section{Acknowledgments}

We would like to thank Jay Armas, Sašo Grozdanov, Nabil Iqbal, Kieran Macfarlane, Watse Sybesma and Lárus Thorlacius for helpful discussions and comments. We are particularly grateful to S. Grozdanov, N. Iqbal and L. Thorlacius for commenting on the manuscript. The work of N. P. was supported by Icelandic Research Fund grant 163422-052 and STFC grant number ST/T000708/1. The work of A.R was supported in part by the Icelandic Research Fund under grant 195970-052 and by the University of Iceland Research Fund.

\section{A Numerical solution and evaluation of operators lifetime}

In this section, remarks on the evaluation of the electric flux are elaborated. The numerical background solution for this geometry can be constructed in the same way as [33] using shooting method. The solution is a one-parameter family characterised by $\mathcal{B} / T^{2}$ which allows us the freedom to choose $r_{h}=1, r_{h}^{2} f^{\prime}\left(r_{h}\right)=1$ (or equivalently $T=1 / 4 \pi$ ). It is also convenient to set $V\left(r_{h}\right)=W\left(r_{h}\right)=0$ which results in the UV boundary metric of the form

$$
\lim _{r \rightarrow \infty} d s^{2}=r^{2}\left(-d t^{2}+v\left(d x^{2}+d y^{2}\right)+w d z^{2}\right)+\frac{d r^{2}}{r^{2}}
$$

Upon rescaling of spatial coordinates $\{d x, d y, d z\} \rightarrow\{d x / \sqrt{v}, d y / \sqrt{v}, d z / \sqrt{w}\}$, we recover the desired background solutions. Note also that the physical magnetic flux is related to the input parameter (that produced the metric in $(\mathrm{A} .1)$ ) by $\mathcal{B}_{\text {physical }}=\mathcal{B}_{\text {input }} / v$. A small caveat of this method is that one cannot find a smooth solution beyond $\mathcal{B}_{\text {input }} \gtrsim \sqrt{3} / 2$ which corresponds to the temperature $T / \sqrt{\mathcal{B}}=\left(4 \pi \sqrt{\mathcal{B}_{\text {input }} / v}\right)^{-1} \approx 0.05$. This is most likely an artifact of the presented numerical method as there exists a smooth solution in the zero temperature limit corresponding to the $A d S_{3} \times \mathbb{R}^{2}$ geometry in the deep IR. We should also note that this is a sufficiently low energy temperature as the entropy becomes sufficiently close to $s \propto T$ obtained from $B T Z \times \mathbb{R}^{2}$ geometry (cf. [27, 33]). The background is generated for $r$ from $\left[1+10^{-3}, 10^{6}\right]$ and varying the (numerical) cutoffs within this order of magnitude does not change the obtained numerical results.

Let us also remark on the numerical value of the renormalised electromagnetic coupling $e_{r}^{-2}=\log \left(\Lambda / r_{h}\right)+\kappa(\Lambda)^{-1}$. This quantity strongly influences both the thermodynamics and low energy spectrum $[27,28,34]$ of the model. In particular a small value of $e_{r}^{-2}$ would result in the speed of sound becoming imaginary [27]. Another way to see that this quantity should be large is to write it in terms of a renormalisation group independent scale $M_{*}$ that denotes the energy scale of a Landau pole [28] i.e. $e_{r}^{-2} \sim \log \left(M_{\star} / T\right)$ where $M_{\star} \gg T$. We take this to be the largest scale in the problem - much larger than the accessible value of $\sqrt{\mathcal{B}} / T$. 


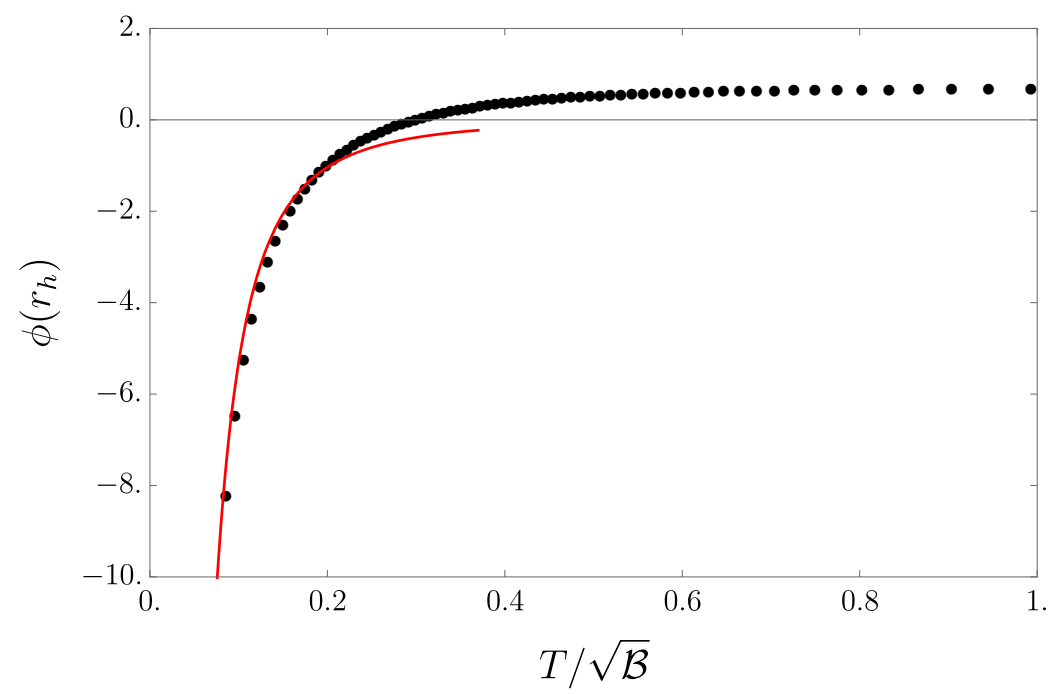

Figure 5. Numerical evaluation of $\phi\left(r_{h}\right)$ in $(2.17)$ as a function of $T / \sqrt{\mathcal{B}}$. The black dots denote the numerical evaluation while the red line denotes the fitting function for small $T / \sqrt{\mathcal{B}}$ as $\phi \approx$ $-(0.008) \frac{\mathcal{B}}{T^{2}} \log \left(5.7 \mathcal{B} / T^{2}\right)$. For high temperatures, the value of $\phi\left(r_{h}\right)$ is approximately constant around 0.69. The value of $\phi\left(r_{h}\right)$ at lowest achievable temperature is at $\phi\left(r_{h}\right)=-23.49$.

Numerical value of the integral for $\phi\left(r_{h}\right)$ in (2.17) is shown in figure 5. For a larger temperature (when $\phi\left(r_{h}\right) \approx \mathcal{O}(1)$ ), the lifetime can be sensibly approximated to be $\tau_{E \|} \approx$ $2 \pi(T / \mathcal{B}) e_{r}^{-2}$. As $T / \sqrt{\mathcal{B}}$ decreases, the lifetime becomes shorter and, if we are to extrapolate the fitting function $\phi \sim \frac{\mathcal{B}}{T^{2}} \log \frac{\mathcal{B}}{T^{2}}$ to even lower temperature where $e_{r}^{-2} \gtrsim \phi$, it will escape the regime of the validity of small $\omega / T, \omega / \sqrt{\mathcal{B}}$ expansions. In this scenario, one shall conclude that there are no long-lived modes that can interfere with the low energy excitations.

\section{B Frobenius analysis in $A d S_{3} \times \mathbb{R}^{2}$ region}

Consider the equation of motion for $\delta B_{x y}$ in the intermediate $A d S_{3} \times \mathbb{R}^{2}$ region:

$$
\delta B_{x y}^{\prime \prime}(r)+\frac{3}{r} \delta B_{x y}^{\prime}(r)+\frac{\omega^{2}}{9 r^{2}} \delta B_{x y}(r)=0
$$

The solution in this region can be obtained via Frobenius method. More precisely, one can change the radial coordinate into $\zeta=3 \omega / r$ and redefine $\delta B_{x y}=\zeta c(\zeta)$. It follows that $c(\zeta)$ is the solution of the Bessel equation of order 1 , which has a regular singular point at $\zeta=0$. The near-boundary $r \rightarrow \infty$, or equivalently $\zeta \rightarrow 0$, akin to the Fefferman-Graham expansion in the usual holographic renormalisation, can be written as

$$
\delta B_{x y}(\zeta)=c_{1}^{M} \mathcal{P}_{1}(\zeta)+\left(c_{2}^{M}+\mathfrak{h} \log \zeta\right) \mathcal{P}_{2}(\zeta)
$$

where $c_{1}^{M}, c_{2}^{M}$ are integration constants and $\mathcal{P}_{i}(\zeta)$ are regular polynomials of the following form

$$
\mathcal{P}_{1}=1+\sum_{n=1}^{\infty} p_{1}^{[n]} \zeta^{n}, \quad \mathcal{P}_{2}=\zeta^{2}\left(1+\sum_{n=1}^{\infty} p_{2}^{[n]} \zeta^{n}\right)
$$


Similar to the usual procedure in the holographic renormalisation [55], all the coefficients $p_{1}^{[n]}, p_{2}^{[n]}, \mathfrak{h}$ except $p_{1}^{[2]}$, which can be set to zero without loss of generality [47], can be obtained recursively. The important piece of information here is the coefficient $\mathfrak{h}=1$ which can be obtained by recursively solving the equation (B.1). Another easy way to see this is to recast (B.1) as the Bessel equation of order 1 as pointed out earlier. Then, using the fact that the Bessel functions $K_{1}(\zeta)$ and $I_{1}(\zeta)$ are two independent solutions of such equation and, for small $\zeta$ they admit the following asymptotic expansions (see e.g. section 3.3 of [56])

$$
I_{1}(\zeta)=\frac{\zeta}{2}+\frac{\zeta^{2}}{16}+\mathcal{O}(\zeta)^{3}, \quad K_{1}(\zeta)=\left(\gamma+\log \frac{\zeta}{2}\right) I_{1}(\zeta)+\frac{1}{\zeta}
$$

will result in the series expansions of the solution in $A d S_{3} \times \mathbb{R}^{2}$ region in (B.2a).

A similar procedure can also be applied for $E^{\perp}$ using eq. (2.25). Substituting $\delta \tilde{B}_{x z}=\zeta^{2} c(\zeta)$, one finds that it obeys the Bessel equation of order 2 whose $\zeta \ll 1$ expansion only yields even power in $\zeta$.

Open Access. This article is distributed under the terms of the Creative Commons Attribution License (CC-BY 4.0), which permits any use, distribution and reproduction in any medium, provided the original author(s) and source are credited.

\section{References}

[1] L.D. Landau and E.M. Lifshitz, Fluid Mechanics, 2nd ed., Butterworth-Heinemann (1987) [DOI].

[2] R.W. Zwanzig, Statistical mechanics of irreversibility, Lectures on Theoretical Physics, Volume 3, Interscience (1961).

[3] D. Forster, Hydrodynamic Fluctuations, Broken Symmetry, and Correlation Functions, Perseus Books, (1995).

[4] S.A. Hartnoll and D.M. Hofman, Locally Critical Resistivities from Umklapp Scattering, Phys. Rev. Lett. 108 (2012) 241601 [arXiv:1201.3917] [INSPIRE].

[5] D. Gaiotto, A. Kapustin, N. Seiberg and B. Willett, Generalized Global Symmetries, JHEP 02 (2015) 172 [arXiv: 1412.5148] [INSPIRE].

[6] S. Grozdanov, D.M. Hofman and N. Iqbal, Generalized global symmetries and dissipative magnetohydrodynamics, Phys. Rev. D 95 (2017) 096003 [arXiv:1610.07392] [INSPIRE].

[7] D. Schubring, Dissipative String Fluids, Phys. Rev. D 91 (2015) 043518 [arXiv:1412.3135] [INSPIRE].

[8] J. Hernandez and P. Kovtun, Relativistic magnetohydrodynamics, JHEP 05 (2017) 001 [arXiv:1703.08757] [INSPIRE].

[9] J. Armas and A. Jain, Magnetohydrodynamics as superfluidity, Phys. Rev. Lett. 122 (2019) 141603 [arXiv: 1808.01939] [InSPIRE].

[10] J. Armas and A. Jain, One-form superfluids \& magnetohydrodynamics, JHEP 01 (2020) 041 [arXiv: 1811.04913] [INSPIRE]. 
[11] W.G. Dixon, Special relativity: the foundation of macroscopic physics, Cambridge University Press Archive (1982).

[12] A.M. Anile, Relativistic fluids and magneto-fluids: With applications in astrophysics and plasma physics, Cambridge University Press (2005).

[13] S.S. Komissarov, A Godunov-type scheme for relativistic magnetohydrodynamics, Mon. Not. Roy. Astron. Soc. 303 (1999) 343.

[14] P.B. Arnold, G.D. Moore and L.G. Yaffe, Transport coefficients in high temperature gauge theories. 1. Leading log results, JHEP 11 (2000) 001 [hep-ph/0010177] [INSPIRE].

[15] J. Pétri, Theory of pulsar magnetosphere and wind, J. Plasma Phys. 82 (2016) 635820502 [arXiv: 1608.04895] [INSPIRE].

[16] R.D. Blandford and R.L. Znajek, Electromagnetic extractions of energy from Kerr black holes, Mon. Not. Roy. Astron. Soc. 179 (1977) 433 [inSPIRE].

[17] S.S. Komissarov, Electrodynamics of black hole magnetospheres, Mon. Not. Roy. Astron. Soc. 350 (2004) 407 [astro-ph/0402403] [INSPIRE].

[18] P. Goldreich and W.H. Julian, Pulsar electrodynamics, Astrophys. J. 157 (1969) 869 [INSPIRE].

[19] T. Wiegelmann and T. Sakurai, Solar Force-free Magnetic Fields, Living Rev. Sol. Phys. 9 (2012) 5 [arXiv:1208.4693] [INSPIRE].

[20] S.E. Gralla and T. Jacobson, Spacetime approach to force-free magnetospheres, Mon. Not. Roy. Astron. Soc. 445 (2014) 2500 [arXiv:1401.6159] [INSPIRE].

[21] G. Compère, S.E. Gralla and A. Lupsasca, Force-Free Foliations, Phys. Rev. D 94 (2016) 124012 [arXiv: 1606.06727] [INSPIRE].

[22] T. Uchida, Theory of force-free electromagnetic fields. I. general theory, Phys. Rev. E 56 (1997) 2181.

[23] C. Thompson and O. Blaes, Magnetohydrodynamics in the extreme relativistic limit, Phys. Rev. D 57 (1998) 3219 [INSPIRE].

[24] S.E. Gralla and N. Iqbal, Effective Field Theory of Force-Free Electrodynamics, Phys. Rev. D 99 (2019) 105004 [arXiv: 1811.07438] [INSPIRE].

[25] P. Glorioso and D.T. Son, Effective field theory of magnetohydrodynamics from generalized global symmetries, arXiv: 1811.04879 [INSPIRE].

[26] B. Benenowski and N. Poovuttikul, Classification of magnetohydrodynamic transport at strong magnetic field, arXiv:1911.05554 [INSPIRE].

[27] S. Grozdanov and N. Poovuttikul, Generalised global symmetries in holography: magnetohydrodynamic waves in a strongly interacting plasma, JHEP 04 (2019) 141 [arXiv: 1707.04182] [INSPIRE].

[28] D.M. Hofman and N. Iqbal, Generalized global symmetries and holography, SciPost Phys. 4 (2018) 005 [arXiv : 1707.08577] [INSPIRE].

[29] D.T. Son and A.O. Starinets, Minkowski space correlators in AdS/CFT correspondence: Recipe and applications, JHEP 09 (2002) 042 [hep-th/0205051] [INSPIRE].

[30] P. Kovtun, D.T. Son and A.O. Starinets, Holography and hydrodynamics: Diffusion on stretched horizons, JHEP 10 (2003) 064 [hep-th/0309213] [INSPIRE]. 
[31] J.F. Fuini and L.G. Yaffe, Far-from-equilibrium dynamics of a strongly coupled non-Abelian plasma with non-zero charge density or external magnetic field, JHEP 07 (2015) 116 [arXiv: 1503.07148] [INSPIRE].

[32] S. Janiszewski and M. Kaminski, Quasinormal modes of magnetic and electric black branes versus far from equilibrium anisotropic fluids, Phys. Rev. D 93 (2016) 025006 [arXiv: 1508.06993] [INSPIRE].

[33] E. D'Hoker and P. Kraus, Magnetic Brane Solutions in AdS, JHEP 10 (2009) 088 [arXiv:0908.3875] [INSPIRE].

[34] S. Grozdanov, A. Lucas and N. Poovuttikul, Holography and hydrodynamics with weakly broken symmetries, Phys. Rev. D 99 (2019) 086012 [arXiv:1810.10016] [INSPIRE].

[35] R.A. Davison and B. Goutéraux, Momentum dissipation and effective theories of coherent and incoherent transport, JHEP 01 (2015) 039 [arXiv: 1411.1062] [INSPIRE].

[36] C.-F. Chen and A. Lucas, Origin of the Drude peak and of zero sound in probe brane holography, Phys. Lett. B $\mathbf{7 7 4}$ (2017) 569 [arXiv:1709.01520] [INSPIRE].

[37] R.A. Davison, S.A. Gentle and B. Goutéraux, Impact of irrelevant deformations on thermodynamics and transport in holographic quantum critical states, Phys. Rev. D 100 (2019) 086020 [arXiv:1812.11060] [INSPIRE].

[38] E. Witten, Multitrace operators, boundary conditions, and AdS/CFT correspondence, hep-th/0112258 [INSPIRE].

[39] M. Berkooz, A. Sever and A. Shomer, 'Double trace' deformations, boundary conditions and space-time singularities, JHEP 05 (2002) 034 [hep-th/0112264] [INSPIRE].

[40] R.A. Davison and A. Parnachev, Hydrodynamics of cold holographic matter, JHEP 06 (2013) 100 [arXiv: 1303.6334] [INSPIRE].

[41] U. Moitra, S.K. Sake and S.P. Trivedi, Near-Extremal Fluid Mechanics, JHEP 02 (2021) 021 [arXiv: 2005.00016] [INSPIRE].

[42] S.S. Gubser and A. Nellore, Ground states of holographic superconductors, Phys. Rev. D 80 (2009) 105007 [arXiv:0908.1972] [inSPIRE].

[43] R.A. Davison, B. Goutéraux and S.A. Hartnoll, Incoherent transport in clean quantum critical metals, JHEP 10 (2015) 112 [arXiv:1507.07137] [INSPIRE].

[44] P.K. Kovtun and A.O. Starinets, Quasinormal modes and holography, Phys. Rev. D 72 (2005) 086009 [hep-th/0506184] [INSPIRE].

[45] E. D'Hoker, P. Kraus and A. Shah, RG Flow of Magnetic Brane Correlators, JHEP 04 (2011) 039 [arXiv: 1012.5072] [INSPIRE].

[46] E. D'Hoker and P. Kraus, Magnetic Field Induced Quantum Criticality via new Asymptotically AdS $S_{5}$ Solutions, Class. Quant. Grav. 27 (2010) 215022 [arXiv:1006.2573] [INSPIRE].

[47] P. Kovtun and A. Starinets, Thermal spectral functions of strongly coupled $N=4$ supersymmetric Yang-Mills theory, Phys. Rev. Lett. 96 (2006) 131601 [hep-th/0602059] [INSPIRE].

[48] M. Abramowitz and I.A. Stegun, Handbook of Mathematical Functions with Formulas, Graphs, and Mathematical Tables, ninth dover printing, tenth gpo printing ed., Dover New York (1964). 
[49] D. Nickel and D.T. Son, Deconstructing holographic liquids, New J. Phys. 13 (2011) 075010 [arXiv: 1009.3094] [INSPIRE].

[50] P. Glorioso, M. Crossley and H. Liu, A prescription for holographic Schwinger-Keldysh contour in non-equilibrium systems, arXiv:1812.08785 [INSPIRE].

[51] J. de Boer, M.P. Heller and N. Pinzani-Fokeeva, Holographic Schwinger-Keldysh effective field theories, JHEP 05 (2019) 188 [arXiv: 1812.06093] [INSPIRE].

[52] S. Bhattacharyya, V.E. Hubeny, S. Minwalla and M. Rangamani, Nonlinear Fluid Dynamics from Gravity, JHEP 02 (2008) 045 [arXiv: 0712.2456] [INSPIRE].

[53] N. Banerjee, J. Bhattacharya, S. Bhattacharyya, S. Dutta, R. Loganayagam and P. Surowka, Hydrodynamics from charged black branes, JHEP 01 (2011) 094 [arXiv:0809.2596] [INSPIRE].

[54] J. Erdmenger, M. Haack, M. Kaminski and A. Yarom, Fluid dynamics of R-charged black holes, JHEP 01 (2009) 055 [arXiv: 0809.2488] [INSPIRE].

[55] S. de Haro, S.N. Solodukhin and K. Skenderis, Holographic reconstruction of space-time and renormalization in the AdS/CFT correspondence, Commun. Math. Phys. 217 (2001) 595 [hep-th/0002230] [INSPIRE].

[56] C. Bender and S. Orszag, Advanced Mathematical Methods for Scientists and Engineers I: Asymptotic Methods and Perturbation Theory, Springer New York, (2013). 